\title{
Effects Radiation and Thermal Diffusion on Hydromagnetic Natural Convection Flow of a Rotating and Chemically Reacting Fluid Past an Infinite Vertical Flat Plate in the Presence of Heat Sink
}

\author{
B. Prabhakar Reddy \\ Department of Mathematics, Geethanjali College of Engineering \& Technology, Keesara, Medchal - 501301, Telangana, India. \\ Department of Mathematics, CNMS, The University of Dodoma, P. Box No.338, Dodoma, Tanzania
}

\begin{abstract}
Effects of radiation and thermal diffusion on an unsteady hydro-magnetic natural convection heat and mass transfer flow of viscous, incompressible, rotating and chemically reacting fluid past an infinite vertical flat plate in the presence heat sink has been presented. The Rosseland approximation is used to describe the radiative heat flux for optically thick fluid in the energy equation. The dimensionless governing partial differential equations of the flow have been solved numerically by using the Ritz finite element method. The effects of various physical parameters involved in the problem under the investigation on the fluid velocity, fluid temperature and the fluid concentration as well as the skin-friction have been examined with the help of graphs and table. It has been found that the fluid temperature and velocity is enhanced in the boundary layer as the radiation parameter increased. The fluid velocity and concentration tends to decrease with increasing Schmidt number and chemical reaction parameter whereas the reverse effect is observed with increasing Soret number. The influence of the physical parameters on the skinfriction shows quite the opposite effect to that of the fluid velocity.
\end{abstract}

Keywords: Natural convection, magnetic field, rotating fluid, radiation parameter, optically thick fluid.

\section{INTRODUCTION}

Natural convection flow of magneto-hydrodynamic fluid has attracted many researchers in view of its numerous applications in the field of geophysics, astrophysics, meteorology, aerodynamics, magneto-hydrodynamic power generators and pumps, boundary layer control energy generators, accelerations, aerodynamic heating, polymer technology, petroleum industry, purification of crude oil, and in material processing such as extrusion, metal forming, continuous casting wire, and glass fiber drawing. Unsteady hydro-magnetic free convection flow with heat flux and accelerated boundary motion was studied by Chandran et. al [1]. Muthucumaraswamy et. al [2] studied the flow past an impulsively started infinite vertical plate in the presence of uniform heat and mass flux at the plate and presented an exact solution using Laplace transform technique. Chaudary and Jain [3] investigated combined effects of heat and mass transfer on MHD free convection flow past an oscillating plate embedded in porous medium. Palani and Abbas [4] studied free convection MHD flow with thermal radiation from an impulsively started vertical plate. Siviah et. al [5] and Das and Jana [6] have studied heat and mass transfer effects on MHD free convection flow past a vertical porous plate with different boundary conditions.

Convection in porous media has its importance in many applications such as geothermal systems, solid matrix heat exchangers, thermal insulations, oil extractions and store of nuclear waste materials. Convection in a porous media can also be applied to underground coal gasification, solar power collectors, iron blast furnaces, wall cooled catalytic reactors, ground water hydrology, energy efficient drying processes, cooling of nuclear fuel in shipping flasks, cooling of electronic equipment and natural convection in earth's crust. Sattar [7] focused on free convection and mass transfer flow through a porous medium past an infinite vertical porous plate with time dependent temperature and concentration. Chamkha [8] investigated thermal radiation and buoyancy effects on hydro-magnetic flow over an accelerating permeable surface with heat source or sink. Acharya et. al [9] presented the magnetic field effects on free convection and mass transfer flow through porous medium with constant suction and constant heat flux. Unsteady MHD convective flow of polar fluids past a vertical moving porous plate in a porous medium was studied by Kim [10]. Chamkha [11] studied the unsteady MHD convective heat and mass transfer past a semi infinite vertical permeable moving plate with heat absorption. The comprehensive review of convective heat transfer mechanism through the porous medium was presented in the book by Donald and Bejan [12]. Ramana and Sastry [13] studied MHD convective flow of a micro-polar fluid past a continuously moving vertical porous plate in the presence of heat generation/absorption. Rajesh and Varma [14] analyzed heat source effects on MHD flow past an exponentially accelerated vertical plate with variable temperature through a porous medium.

Combined heat and mass transfer flows with chemical reaction are of important in many processes and have therefore received a considerable amount of attention in recent years. Possible applications of this type of flow can be found in many industries. The rate of reaction depends on the concentration of species itself. The effects of chemical reaction on heat and mass transfer along a wedge with heat source and convection in the presence of suction or injection was investigated by Kandasamy et. al [15]. Seddeek et. al [16] analyzed the effects of chemical reaction and variable 
viscosity on hydro-magnetic mixed convection heat and mass transfer for hiemenz flow through porous medium with radiation. Muthucumaraswamy and Janakiraman [17] presented mass transfer effects on isothermal vertical oscillating plate in the presence of chemical reaction. Mahdy [18] investigated the effects of chemical reaction and heat generation or absorption on double diffusive convection from a vertical truncated cone in porous media with variable viscosity. Alharbi et. al [19] studied heat and mass transfer in MHD visco-elastic fluid through a porous medium over a stretching sheet with chemical reaction. Magnetic field effects on heat and mass transfer of hydro-magnetic flow past a vertical oscillating plate in the presence of chemical reaction were investigated by Senapati and Dhal [20]. Sekhar and Reddy [21] analyzed chemical reaction effects on MHD free convection oscillatory flow past a porous plate with viscous dissipation and heat sink.

The study of magneto-hydrodynamic with heat and mass transfer in the presence of radiation and rotation has applications in space science, engineering fluid dynamics, geophysics, astrophysical flows, solar power technology and stellar structures etc. Agarwal et. al [22] presented the effects of Hall current on hydro-magnetic free convection with mass transfer in a rotating fluid. Bestman and Adjepong [23] studied unsteady hydro-magnetic free convection flow with radiative heat transfer in a rotating fluid. Radiation effects on MHD mixed free-fixed convection flow past a semi infinite moving vertical plate for high temperature differences was investigated by Azzam [24]. Cookey et. al [25] focused on the influence of viscous dissipation and radiation on unsteady MHD free convection flow past an infinite heated vertical plate in a porous medium with time dependent suction. Mbeledogu and Ogulu [26] studied heat and mass transfer of an unsteady MHD natural convection flow of a rotating fluid past a vertical porous flat plate in the presence of radiative heat transfer. Shanker and Gnaneshwar [27] analyzed radiation effects on MHD flow past an impulsively started infinite vertical plate through a porous medium with variable temperature and mass diffusion. Thermal radiation effects on unsteady MHD free convection flow past a vertical plate with temperature dependent suction was presented by Mahmoud [28]. Ahmed and Sarmah [29] investigated thermal radiation effects on a transient MHD flow with mass transfer past an impulsively fixed infinite vertical plate. The effects of thermal radiation on unsteady mixed convection flow and heat transfer over a porous stretching surface in a porous medium was investigated by Mukhopadhyay [30]. Sharma and Deka [31] analyzed thermal radiation and oscillating plate temperature effects on unsteady MHD flow past a semi infinite porous vertical plate in the presence of chemical reaction. Muralidharan and Muthucumaraswamy [32] investigated radiation effects on linearly accelerated plate with variable mass diffusion in the presence of magnetic field.

However, in the literature we found less attention was paid on unsteady hydro-magnetic heat and mass transfer flow of viscous, incompressible, rotating Boussinesq fluid past an infinite vertical porous plate with thermal diffusion (Soret) effect under the different conditions, even though this situation has become increasingly important in engineering and industrial applications. For example, the Soret effect has been utilized for isotopes separation in the mixture between the gases with light molecular weight (like $\mathrm{H}, \mathrm{He}$ ) and medium molecular weight (like $\mathrm{O}, \mathrm{NH}_{3}$ ). In separation such gases, the Soret effect was found to be magnitude that cannot be neglected. Hence, due to its important industrial and engineering applications, it is proposed to investigate radiation and thermal diffusion effects on unsteady hydromagnetic natural convection heat and mass transfer flow of viscous, incompressible, rotating and chemically reacting fluid past an infinite vertical flat porous plate in the presence of heat sink. The Ritz finite element method has been adopted to solve the governing equations of the problem, which is more economical from the computational point of view. The influence of the physical parameters encountered in the problem under the investigation on the flow has been presented through the graphs and table and then discussed.

\section{MATHEMATICAL MODEL}

We consider an unsteady hydro-magnetic natural convection flow of an incompressible, electrically conducting, viscous Boussinesq fluid past an infinite vertical porous flat plate with radiation and chemical reaction. In the coordinate system, $x^{\prime}$ - axis taken along the plate in the upward direction and $y^{\prime}-$ axis is taken normal to it. A uniform magnetic field of strength $B_{0}$ applied in the direction of the flow fixed relative to the plate. We assume that induced magnetic field is neglected in comparison to the applied magnetic field. The plate is infinite in length, so all the field quantities become functions of the space coordinate $y^{\prime}$ and time $t^{\prime}$. Initially $\left(t^{\prime} \leq 0\right)$, the plate and the fluid are at the same temperature $T_{\infty}^{\prime}$ and concentration $C_{\infty}^{\prime}$. At time $t^{\prime}>0$, the fluid starts moving in its own plane with velocity $U_{0}$ and rotates with angular velocity $\Omega$ as in [23], its temperature raised to $T_{w}^{\prime}$ and the concentration level at the plate raised to $C_{w}^{\prime}$. Further, we assume that no applied voltage present which means no electric field present and viscous dissipation heating is absent in the energy equation. With these assumptions and those usually associated with the Boussinesq approximations, the proposed governing equations of the flow are:

$$
\begin{aligned}
& \frac{\partial u^{\prime}}{\partial t^{\prime}}-2 \Omega^{\prime} v^{\prime}=v \frac{\partial^{2} u^{\prime}}{\partial y^{\prime 2}}-\frac{\sigma B_{0}^{2} u^{\prime}}{\rho}-\frac{v u^{\prime}}{K^{\prime}}+g \beta\left(T^{\prime}-T_{\infty}^{\prime}\right)+ \\
& g \beta^{*}\left(C^{\prime}-C_{\infty}^{\prime}\right) \\
& \frac{\partial v^{\prime}}{\partial t^{\prime}}-2 \Omega^{\prime} u^{\prime}=v \frac{\partial^{2} v^{\prime}}{\partial y^{\prime 2}}-\frac{\sigma \mu^{2} H_{0}^{2} v^{\prime}}{\rho} \\
& \rho c_{p} \frac{\partial T^{\prime}}{\partial t^{\prime}}=k \frac{\partial^{2} T^{\prime}}{\partial y^{\prime 2}}-\frac{\partial q_{r}^{*}}{\partial y^{\prime}}-Q_{0}^{\prime}\left(T^{\prime}-T_{\infty}^{\prime}\right) \\
& \frac{\partial C^{\prime}}{\partial t^{\prime}}=D_{m} \frac{\partial^{2} C^{\prime}}{\partial y^{\prime 2}}+\frac{D_{m} K_{T}}{T_{m}}\left(\frac{\partial^{2} T^{\prime}}{\partial y^{\prime 2}}\right)-k_{r}^{\prime}\left(C^{\prime}-C_{w}^{\prime}\right)
\end{aligned}
$$


with corresponding initial and boundary condition are:

$$
\begin{array}{lll}
t^{\prime} \leq 0 ; & u^{\prime}=0, v^{\prime}=0, T^{\prime}=T_{\infty}^{\prime}, C^{\prime}=C_{\infty}^{\prime} & \forall y^{\prime} \\
t^{\prime}>0 ; & u^{\prime}=U_{0}, v^{\prime}=0, T^{\prime}=T_{w}^{\prime}, C^{\prime}=C_{w}^{\prime} & \text { on } y^{\prime}=0 \\
& u^{\prime}=0, v^{\prime}=0, T^{\prime}=T_{\infty}^{\prime}, C^{\prime}=C_{\infty}^{\prime} & \text { as } y^{\prime} \rightarrow \infty
\end{array}
$$

where

$u^{\prime}, v^{\prime}, g, \beta, \beta^{*}, T^{\prime}, T_{\infty}^{\prime}, T_{w}^{\prime}, C^{\prime}, C_{\infty}^{\prime}, C_{w}^{\prime}, Q_{0}^{\prime}, k, v, \mu, \rho, \sigma, c_{p}, q_{r}^{\prime}$, $k_{r}^{\prime}, T_{m}, K_{T}, D_{m}$ and $t^{\prime}$ are, respectively, velocity components in $x^{\prime}$ and $y^{\prime}$ - directions, acceleration due gravity, volumetric coefficient of thermal expansion, volumetric coefficient of concentration expansion, temperature of the fluid, free stream temperature, surface temperature, concentration of the fluid, free stream concentration, surface concentration, heat absorption coefficient, thermal conductivity, kinematic viscosity, fluid viscosity, fluid density, electrical conductivity, specific heat at constant pressure, radiative heat flux in $x^{\prime}$ - direction, chemical reaction constant, mean fluid temperature, thermal diffusion ratio, mass diffusivity and time.

The radiative heat flux $q_{r}^{*}$ on the basis of the Rosseland approximation expressed as:

$$
q_{r}^{*}=-\frac{4 \sigma^{*}}{3 k^{*}} \frac{\partial T^{\prime 4}}{\partial y^{\prime}}
$$

where $\sigma^{*}$ is the Stefan-Boltzmann constant and $k^{*}$ is the mean absorption coefficient. It is assumed that the temperature differences within the flow are sufficiently small such that the term $T^{\prime 4}$ is expressed as the linear function of temperature. Therefore, expanding $T^{\prime 4}$ about a free stream temperature $T_{\infty}^{\prime}$, using Taylor series expansion and neglecting higher order terms, one obtain

$T^{\prime 4} \square T_{\infty}^{\prime 4}+4 T_{\infty}^{\prime 3}\left(T^{\prime}-T_{\infty}^{\prime}\right) \square 4 T_{\infty}^{\prime 3} T^{\prime}-3 T_{\infty}^{\prime 4}$

Using equation (7) in equation (6), we obtain

$q_{r}^{*}=-\frac{16 \sigma^{*} T_{\infty}^{\prime 3}}{3 k^{*}} \frac{\partial T^{\prime}}{\partial y^{\prime}}$

Using Eq. (8) into Eq. (3), we arrive at the energy equation

$\rho C_{p} \frac{\partial T^{\prime}}{\partial t^{\prime}}=k \frac{\partial^{2} T^{\prime}}{\partial y^{\prime 2}}+\frac{16 \sigma^{*} T_{\infty}^{\prime 3}}{3 k^{*}} \frac{\partial^{2} T^{\prime}}{\partial y^{\prime 2}}-Q_{0}^{\prime}\left(T^{\prime}-T_{\infty}^{\prime}\right)$

We now introduce the following non-dimensional quantities and parameters

$$
\begin{aligned}
& t^{\prime}=\frac{t v}{U_{0}^{2}}, y^{\prime}=\frac{y v}{U_{0}}, u^{\prime}=u U_{0}, v^{\prime}=v U_{0}, S_{c}=\frac{v}{D_{m}}, P_{r}=\frac{\mu c_{p}}{k}, \\
& \Omega^{\prime}=\frac{U_{0}^{2} \Omega}{v}, M^{2}=\frac{\mu \sigma B_{0}^{2} v}{\rho U_{0}^{2}}, K^{\prime}=\frac{v^{2} K}{U_{o}^{\prime 2}}, \theta=\frac{T^{\prime}-T_{w}^{\prime}}{T_{w}^{\prime}-T_{\infty}^{\prime}}, \\
& \phi=\frac{C^{\prime}-C_{w}^{\prime}}{C_{w}^{\prime}-C_{\infty}^{\prime}}, G_{r}=\frac{g \beta v\left(T^{\prime}-T_{\infty}^{\prime}\right)}{U_{0}^{3}}, G_{m}=\frac{g \beta^{*} v\left(C^{\prime}-C_{\infty}^{\prime}\right)}{U_{0}^{3}},
\end{aligned}
$$

$$
\begin{aligned}
& H=M^{2}+\frac{1}{K}, Q_{H}=\frac{Q_{0}^{\prime} v}{U_{0}^{2}}, S_{r}=\frac{D_{m} K_{T}\left(T_{w}^{\prime}-T_{\infty}^{\prime}\right)}{v T_{m}\left(C_{w}^{\prime}-C_{\infty}^{\prime}\right)}, \\
& R=\frac{16 \sigma^{*} T_{\infty}^{\prime 3}}{3 k k^{*}}, F=\frac{P_{r}}{1+R}, L=F Q_{H} .
\end{aligned}
$$

In view of equation (10), equations (1),(2),(4),(5) and (9) reduced to the following dimensionless form:

$$
\begin{aligned}
& \frac{\partial u}{\partial t}-2 \Omega v=\frac{\partial^{2} u}{\partial y^{2}}-H u+G_{r} \theta+G_{m} \phi \\
& \frac{\partial v}{\partial t}-2 \Omega u=\frac{\partial^{2} v}{\partial y^{2}}-M^{2} v
\end{aligned}
$$

$$
F \frac{\partial \theta}{\partial t}=\frac{\partial^{2} \theta}{\partial y^{2}}-L \theta
$$

$\frac{\partial \phi}{\partial t}=\frac{1}{S_{c}} \frac{\partial^{2} \phi}{\partial y^{2}}+S_{r}\left(\frac{\partial^{2} \theta}{\partial y^{2}}\right)-k_{r} \phi$

With non-dimensional initial and boundary conditions:

$$
\begin{aligned}
t \leq 0: u=0, v=0, \theta=0, \phi=0 & \forall y \\
t>0: u=u_{0}, v=0, \theta=1, \phi=1 & \text { at } y=0 \\
u \rightarrow 0, v \rightarrow 0, \theta \rightarrow 0, \phi \rightarrow 0 & \text { as } y \rightarrow \infty
\end{aligned}
$$

\section{METHOD OF SOLUTION}

By applying the Ritz finite element method to the Eq. (11) over the two-nodded linear element $(e),\left(y_{j} \leq y \leq y_{k}\right)$ is

$$
J^{(e)}(u)=\frac{1}{2} \int_{z_{j}}^{z_{k}}\left\{\left(\frac{\partial u^{(e)}}{\partial y}\right)^{2}+H u^{(e)^{2}}+2 u^{(e)} \frac{\partial u^{(e)}}{\partial t}-2 u^{(e)} \Delta\right\} d y=
$$

minimum

where $\Delta=2 \Omega v+G_{r} \theta+G_{m} \phi$.

Let $u^{(e)}=\psi_{j}(y) u_{j}(t)+\psi_{k}(y) u_{k}(t)=\psi_{j} u_{j}+\psi_{k} u_{k}$ be the linear piecewise approximation solution over the element $(e)$ $\left(y_{j} \leq y \leq y_{k}\right)$. We obtain the element equation with $l^{(e)}=y_{k}-y_{j}$ is

$\frac{1}{l^{(e)}}\left[\begin{array}{cc}1 & -1 \\ -1 & 1\end{array}\right]\left[\begin{array}{l}u_{j} \\ u_{k}\end{array}\right]+H \frac{l^{(e)}}{6}\left[\begin{array}{ll}2 & 1 \\ 1 & 2\end{array}\right]\left[\begin{array}{l}u_{j} \\ u_{k}\end{array}\right]+\frac{l^{(e)}}{6}\left[\begin{array}{ll}2 & 1 \\ 1 & 2\end{array}\right]\left[\begin{array}{c}u_{j}^{\bullet} \\ u_{k}^{\bullet}\end{array}\right]-$ $\Delta \frac{l^{(e)}}{2}\left[\begin{array}{l}1 \\ 1\end{array}\right]=0$

Where the prime and the dot denotes the differentiation with respect to $y$ and $t$. In order to get the difference equation at the node $i$, we write the element equations for two consecutive elements $y_{i-1} \leq y \leq y_{i}$ and $y_{i} \leq y \leq y_{i+1}$, assembling these two element equations, one get 


$$
\begin{gathered}
\frac{1}{l^{(e)}}\left[\begin{array}{ccc}
1 & -1 & 0 \\
-1 & 2 & -1 \\
0 & -1 & 1
\end{array}\right]\left[\begin{array}{c}
u_{i-1} \\
u_{i} \\
u_{i+1}
\end{array}\right]+H \frac{l^{(e)}}{6}\left[\begin{array}{ccc}
2 & 1 & 0 \\
1 & 4 & 1 \\
0 & 1 & 2
\end{array}\right]\left[\begin{array}{c}
u_{i-1} \\
u_{i} \\
u_{i+1}
\end{array}\right] \\
+\frac{l^{(e)}}{6}\left[\begin{array}{ccc}
2 & 1 & 0 \\
1 & 4 & 1 \\
0 & 1 & 2
\end{array}\right]\left[\begin{array}{c}
u_{i-1}^{\bullet} \\
u_{i}^{\bullet} \\
u_{i+1}^{\bullet}
\end{array}\right]=\Delta \frac{l^{(e)}}{2}\left[\begin{array}{l}
1 \\
2 \\
1
\end{array}\right]
\end{gathered}
$$

Put row corresponding to the node $i$ to zero in Eq. (17), the following difference schemes with $l^{(e)}=h$ are obtained:

$$
\begin{gathered}
\frac{6}{h^{2}}\left(-u_{i-1}+2 u_{i}-u_{i+1}\right)+H\left(u_{i-1}+4 u_{i}+u_{i+1}\right) \\
+\left(u_{i-1}^{\bullet}+4 u_{i}^{\bullet}+u_{i+1}^{\bullet}\right)=6 \Delta
\end{gathered}
$$

Applying the trapezoidal rule to Eq. (18), the following system of equations are obtained in Crank-Nicholson method:

$$
\begin{aligned}
& A_{1} u_{i-1}^{j+1}+A_{2} u_{i}^{j+1}+A_{1} u_{i+1}^{j+1}=A_{3} u_{i-1}^{j}+A_{4} u_{i}^{j}+A_{3} u_{i+1}^{j}+\Omega_{1 i}^{j} \\
& B_{1} v_{i-1}^{j+1}+B_{2} v_{i}^{j+1}+B_{1} v_{i+1}^{j+1}=B_{3} v_{i-1}^{j}+B_{4} v_{i}^{j}+B_{3} v_{i+1}^{j}+\Omega_{2 i}^{j} \\
& C_{1} \theta_{i-1}^{j+1}+C_{2} \theta_{i}^{j+1}+C_{1} \theta_{i+1}^{j+1}=C_{3} \theta_{i-1}^{j}+C_{4} \theta_{i}^{j}+C_{3} \theta_{i+1}^{j} \\
& D_{1} \phi_{i-1}^{j+1}+D_{2} \phi_{i}^{j+1}+D_{1} \phi_{i+1}^{j+1}=D_{3} \phi_{i-1}^{j}+D_{4} \phi_{i}^{j}+D_{3} \phi_{i+1}^{j}+\Omega_{3 i}^{j}
\end{aligned}
$$

Where

$$
\begin{aligned}
& A_{1}=1-3 r+\frac{1}{2} r H h^{2} ; A_{2}=4+6 r+2 r H h^{2} \\
& A_{3}=1+3 r-\frac{1}{2} r H h^{2} ; A_{4}=4-3 r-\frac{1}{2} r H h^{2} \\
& B_{1}=1-3 r+\frac{1}{2} r M^{2} h^{2} ; B_{2}=4+6 r+2 r M^{2} h^{2} \\
& B_{3}=1+3 r-\frac{1}{2} r M^{2} h^{2} ; B_{4}=4-3 r-\frac{1}{2} r M^{2} \rightarrow h^{2} \\
& C_{1}=F-3 r+\frac{1}{2} r Q_{H} F h^{2} ; C_{2}=4 F+6 r+2 r Q_{H} F h^{2} \\
& C_{3}=F+3 r-\frac{1}{2} r Q_{H} F h^{2} ; C_{4}=F-3 r-\frac{1}{2} r Q_{H} F h^{2} \\
& D_{1}=S_{c}-3 r+\frac{1}{2} r k_{r} S_{c} h^{2} ; D_{2}=4 S_{c}+6 r+2 r k_{r} S_{c} h^{2} \\
& D_{3}=S_{c}+3 r-\frac{1}{2} r k_{r} S_{c} h^{2} ; D_{4}=4 S_{c}-6 r-2 r k_{r} S_{c} h^{2} \\
& \Omega_{1 i}^{j}=6 k\left(2 \Omega v_{i}^{j}+G_{r} \theta_{i}^{j}+G_{m} \phi_{i}^{j}\right) ; \\
& \Omega_{2 i}^{j}=12 k \Omega u_{i}^{j} ; \Omega_{3 i}^{j}=6 r S_{c} S_{r}\left(\theta_{i-1}^{j}-2 \theta_{i}^{j}+\theta_{i+1}^{j}\right)
\end{aligned}
$$

Here $r=h / k^{2}$ and $h, k$ are the mesh sizes along $y-$ direction and time $t-$ direction, respectively. Index $i$ refers to the space and $j$ refers to the time. In equations (19)-(22), taking $i=1(1) n$ and using initial and boundary conditions given in equation (15), the following tri-diagonal system of equations are obtained:

$$
\begin{aligned}
& A u=A^{\prime} \\
& B v=B^{\prime} \\
& C \theta=C^{\prime} \\
& D \phi=D^{\prime}
\end{aligned}
$$

where $A, B, C$ and $D$ are tri-diagonal matrices of order- $n$ whose elements are given by

$$
\begin{array}{ll}
a_{i-1, i}=A_{1} ; b_{i-1, i}=B_{1} ; c_{i-1, i}=C_{1} ; d_{i-1, i}=D_{1} & \text { at } i=2(1) n \\
a_{i i}=A_{2} ; b_{i i}=B_{2} ; c_{i i}=C_{2} ; d_{i i}=D_{2} & \text { at } i=1(1) n \\
a_{i, i-1}=A_{1} ; b_{i, i-1}=B_{1} ; c_{i, i-1}=C_{1} ; d_{i, i-1}=D_{1} & \text { at } i=2(1) n
\end{array}
$$

Here $u, v, \theta, \phi$ and $A^{\prime}, B^{\prime}, C^{\prime}, D^{\prime}$ are column matrices having $n$ - components $u_{i}^{j+1}, v_{i}^{j+1}, \theta_{i}^{j+1}, \phi_{i}^{j+1}$ and $u_{i}^{j}, v_{i}^{j}, \theta_{i}^{j}, \phi_{i}^{j}$ respectively. The Gauss-Seidal iteration scheme is employed to solve the system of equations given in Eq. (23). Numerical results for the velocity, temperature and the concentration profiles are obtained by using $C$ - program. The boundary condition $y \rightarrow \infty$ is approximated by $y_{\max }=5$, which is sufficiently large for the velocity to approach convergence criterion. The computations are carried out until the steady state is reached. The steady state solution is assumed to have been reached when the difference between any two time steps is less than $10^{-5}$ at all nodal points. To judge the convergence of the method, computations are carried out by making small changes in the space and time directions by running the same program, no significant change was observed in the values of $u, v, \theta$ and $\phi$. Hence, we conclude that the Ritz finite element method is convergent and stable.

The skin-friction coefficient $(\tau)$ in non-dimensional form at the plate is given by $\tau=-\left(\frac{\partial u}{\partial y}\right)_{y=0}$

\section{RESULTS AND DISCUSSION}

To investigate the physical significance of various physical parameters on the flow, numerical calculations have been carried out for the fluid velocity, fluid temperature, and the fluid concentration and presented them graphically whereas the numerical values of skin-friction presented in a tabular form. These results show the effects of material parameters on the fluid velocity, fluid temperature and fluid concentration as well as the skin-friction.

Figure 1illustrates the effects of the magnetic field parameter $M$ on the fluid velocity. The velocity curves show that the rate of transport is remarkably reduced with increasing values of the magnetic parameter. This result agrees qualitatively with the expectations, since the magnetic field exerts a retarding effect on the natural convection flow. The variation of the fluid velocity with increasing values of the radiation parameter $R$ is presented in Fig.2. It is seen that the fluid velocity increases with increasing values of the radiation parameter. It may be noted that large values of $R$ corresponds to an increased dominance of conduction over the radiation thereby increasing buoyancy force and thickness of the 
momentum boundary layer. Figure 3 shows that the effect of the Prandtl number $P_{r}$ on the fluid velocity for four different physically realistic values of $P_{r}=0.71,1.0,7.0$ and 100.0 which, corresponds to air, electrolytic solution, water and engine oil, respectively. It is noticed that an increase in the Prandtl number tends to decrease the fluid velocity. This result can happen due to the fact that large of $P_{r}$ leads to increase of fluid viscosity and decreases thermal conductivity, which makes the fluid thick and causes a decrease in the fluid velocity. The effects of the heat absorption parameter $Q_{H}$ on the fluid velocity are shown in Fig. 4. It is observed that increasing values of $Q_{H}$ decreases the fluid velocity in the boundary layer. This result is expected since the presence of heat sink in the boundary layer absorbs energy, which in turn causes a decrease in the fluid temperature. This decrease in the temperature produces a decrease in the fluid velocity due to the buoyancy effect, which couples the flow and thermal fields. Figure 5 illustrates the effect of thermal Grashof number $G_{r}$ on the fluid velocity. It can be seen that the velocity of the fluid increases with increasing values of thermal Grashof number $G_{r}$. It may be noted that buoyancy force enhances fluid velocity and increases the boundary layer thickness with increasing values of $G_{r}$. Also, it is observed that distinctive peaks in the velocity profiles occur in the fluid adjacent to the wall for large values of $G_{r}$. The presence of the peaks indicates that the maximum values of the fluid velocity occurs in the body of the fluid close to the surface and not at the surface. Figure 6 plotted to show the effects of the mass Grashof number $G_{m}$ on the fluid velocity. It is observed that the effect of $G_{m}$ on the fluid velocity is same as that of $G_{r}$. This result can be achieved by comparing Figs.5 and 6.The variation of the fluid velocity with dimensionless permeability parameter $K$ is presented in the Fig.7. It is noticed that the fluid velocity increases with increasing values of dimensionless permeability parameter $K$. It may be noted when the holes of the porous medium are very large so that the resistance of the medium may be neglected. Figure 8 depicts the effect of rotation parameter $\Omega$ on the fluid velocity. It is observed that the fluid velocity increases with increasing values of the rotation parameter. Figure 9 shows the effect of Schmidt number $S_{c}$ on the fluid velocity for four physically realistic values of $S_{c}=0.22,0.60,0.78$ and 1.0, which corresponds to hydrogen, water-vapour, ammonia and methanol, respectively. This figure clearly indicates that the fluid velocity decreases with increasing values of Schmidt number $S_{c}$, due to the decrease in the molecular diffusivity, which result in a decrease in the concentration and velocity boundary layer thickness. The influence of Soret number $S_{r}$ on the fluid velocity is presented in Fig. 10. It clearly seen that the fluid velocity is completely opposite to that of Schmidt number. This result can be clearly seen by comparing Figs. 9 and 10. Figure 11 demonstrates the effects of chemical reaction rate $k_{r}$ on the fluid velocity. It is observed that fluid velocity increases with increasing chemical reaction rate.

The effects of heat absorption parameter $Q_{H}$ on the fluid temperature are shown in Fig.12. It can be seen that the there is a fall in the temperature $\theta$ in the boundary layer as the heat absorption parameter increased. It may be noted that the presence of heat sink in the boundary layer absorbs energy, which in turn causes a decrease in the fluid temperature. Figure 13 shows the effects of Prandtl number $P_{r}$ on the fluid temperature. It is found that that the fluid temperature decreases in the boundary layer with increasing Prandtl number, due to decrease in the thermal boundary layer thickness and more uniform temperature distribution across the boundary layer. The influence of the radiation parameter $R$ on the fluid temperature is depicted in Fig. 14. It is observed that the temperature $\theta$ is enhanced in the boundary layer as the radiation parameter increased. It may be noted that large values of $R$ correspond to an increased dominance of conduction over radiation thereby increasing the thickness of the thermal boundary layer.

The variation of the fluid concentration $\phi$ with increasing values of the Schmidt number $S_{c}$ is shown in Fig. 15. It is noticed that fluid concentration decreases in the boundary layer as the Schmidt number increases. Physically, increase of $S_{c}$ tends to decrease the molecular diffusivity which results a decrease of concentration boundary layer. Figure 16 presents the effect of the Soret number $S_{r}$ on the fluid concentration. It is observed that there is a marked effect of increasing values of Soret number $S_{r}$ on the fluid concentration in the boundary layer. This implies that increasing values of $S_{r}$ tends to increase in the fluid concentration. The effects of the chemical reaction rate $k_{r}$ on the fluid concentration are presented in Fig. 17. We found that increasing values of $k_{r}$ tends to decrease the concentration of the species in the boundary layer. Physically, this is due to the fact that large values of $k_{r}$ reduces the solutal boundary layer thickness and increase the mass transfer.

The effects of $P_{r}, Q_{H}, S_{c}, k_{r}, M, R, S_{r}, K, \Omega, G_{r}$ and $G_{m}$ on the skin friction $(\tau)$ are presented in table 1 . Table 1 reveals that Prandtl number, heat absorption parameter, Schmidt number, chemical reaction parameter and magnetic parameter tends to increase the skin friction whereas radiation parameter, Soret number, porosity parameter, rotation parameter, thermal Grashof number and mass Grashof number tends to decrease the skin friction. 


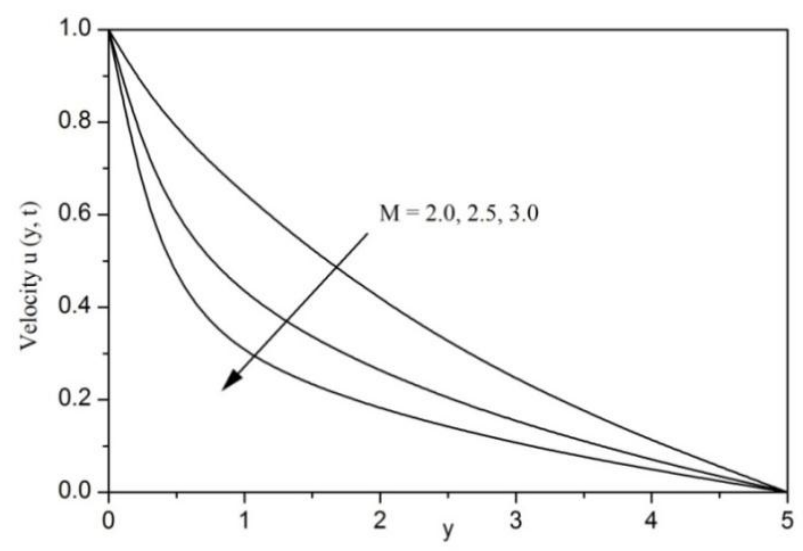

Fig. 1: Effect of magnetic parameter $M$ on the fluid velocity when $G_{r}=2.0, G_{m}=2.0$, $P_{r}=0.71, S_{c}=0.22, R=1.0, Q_{H}=0.5, S_{r}=1.0, k_{r}=0.5, K=1.0, \Omega=1.0$ at $t=1.0$.

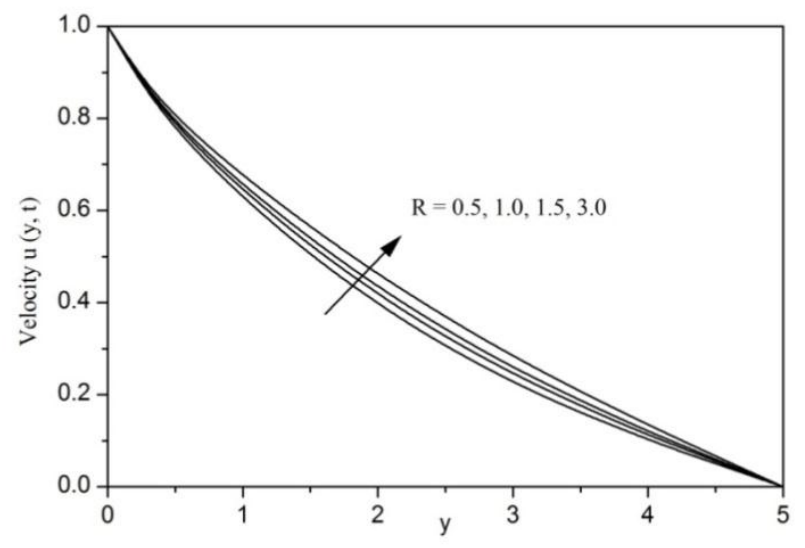

Fig. 2: Effect of radiation parameter $R$ on the fluid velocity when $G_{r}=2.0, G_{m}=2.0$, $P_{r}=0.71, S_{c}=0.22, Q_{H}=0.5, S_{r}=1.0, k_{r}=0.5, K=1.0, M=2.0, \Omega=1.0$ at $t=1.0$.

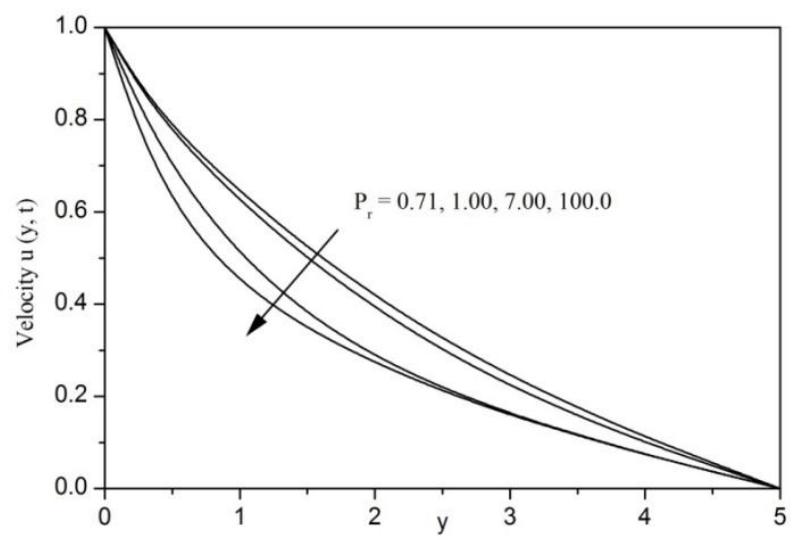

Fig. 3: Effect of Prandtl number $P_{r}$ on the fluid velocity when $G_{r}=2.0, G_{m}=2.0$, $S_{c}=0.22, R=1.0, Q_{H}=0.5, S_{r}=1.0, k_{r}=0.5, K=1.0, M=2.0, \Omega=1.0$ at $t=1.0$. 


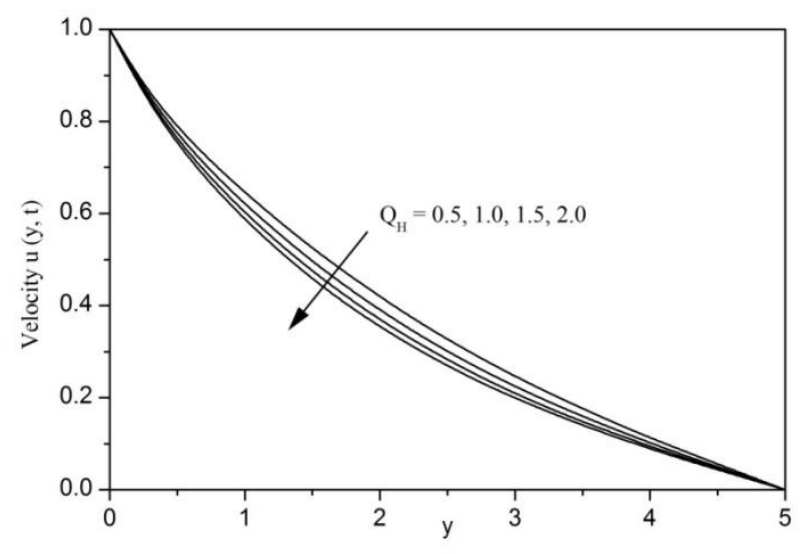

Fig. 4: Effect of heat absorption parameter $Q_{H}$ on the fluid velocity when $G_{r}=2.0, G_{m}=2.0$, $P_{r}=0.71, S_{c}=0.22, R=1.0, S_{r}=1.0, k_{r}=0.5, K=1.0, M=2.0, \Omega=1.0$ at $t=1.0$.

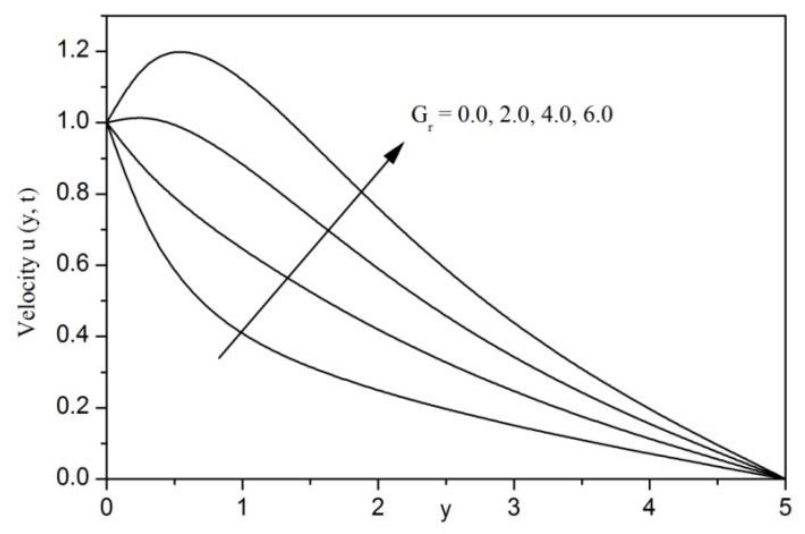

Fig. 5: Effect of thermal Grashof number $G_{r}$ on the fluid velocity when $G_{m}=2.0, P_{r}=0.71$, $S_{c}=0.22, R=1.0, Q_{H}=0.5, S_{r}=1.0, k_{r}=0.5, K=1.0, M=2.0, \Omega=1.0$ at $t=1.0$.

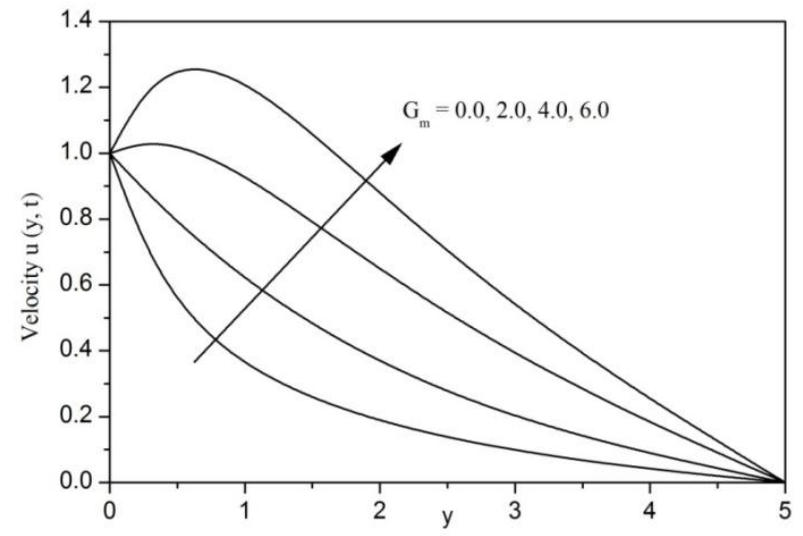

Fig. 6: Effect of mass Grashof number $G_{m}$ on the fluid velocity when $G_{r}=2.0, P_{r}=0.71$, $S_{c}=0.22, R=1.0, Q_{H}=0.5, S_{r}=1.0, k_{r}=0.5, K=1.0, M=2.0, \Omega=1.0$ at $t=1.0$. 


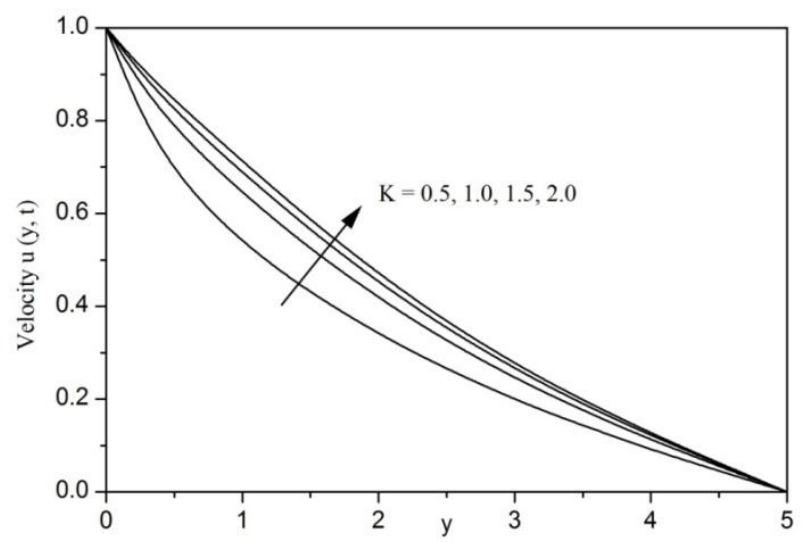

Fig. 7: Effect of permeability parameter $K$ on the fluid velocity when $G_{r}=2.0, G_{m}=2.0$, $P_{r}=0.71, S_{c}=0.22, R=1.0, Q_{H}=0.5, S_{r}=1.0, k_{r}=0.5, M=2.0, \Omega=1.0$ at $t=1.0$.

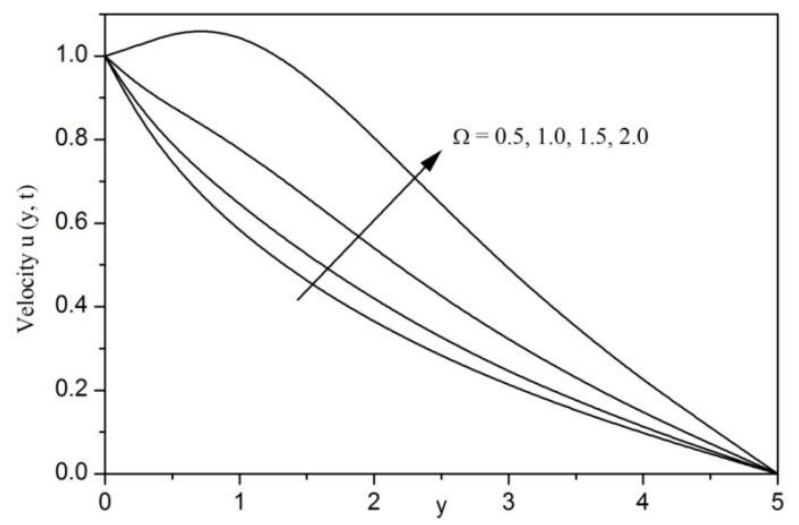

Fig. 8: Effect of rotation parameter $\Omega$ on the fluid velocity when $G_{r}=2.0, G_{m}=2.0$, $P_{r}=0.71, S_{c}=0.22, R=1.0, Q_{H}=0.5, S_{r}=1.0, k_{r}=0.5, K=1.0, M=2.0$ at $t=1.0$.

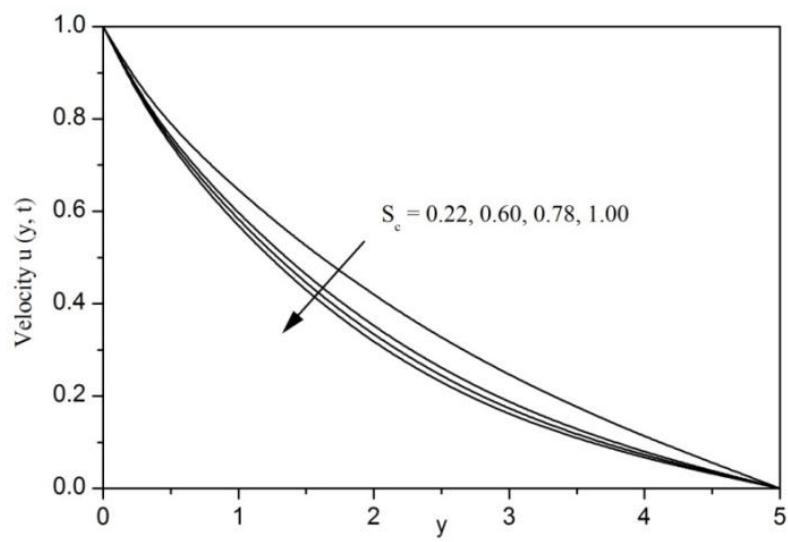

Fig. 9: Effect of Schmidt number $S_{c}$ on the fluid velocity when $G_{r}=2.0, G_{m}=2.0$, $P_{r}=0.71, R=1.0, Q_{H}=0.5, S_{r}=1.0, k_{r}=0.5, K=1.0, M=2.0, \Omega=1.0$ at $t=1.0$. 


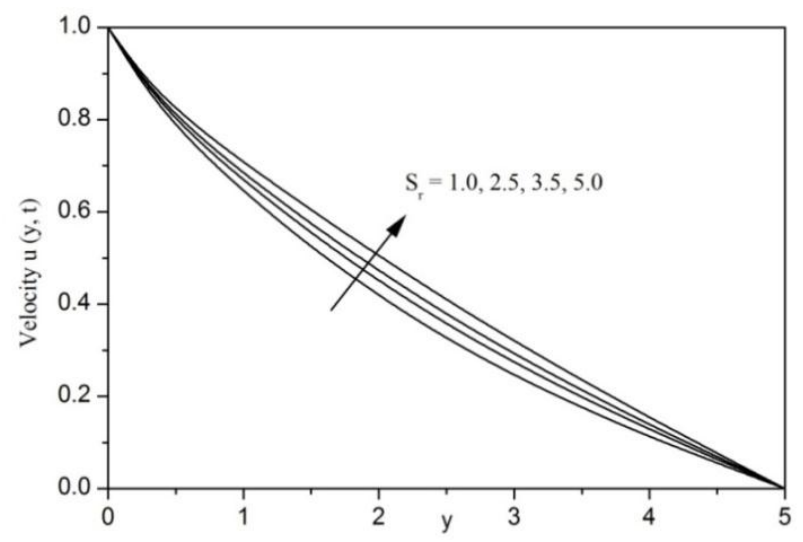

Fig. 10: Effect of Soret number $S_{r}$ on the fluid velocity when $G_{r}=2.0, G_{m}=2.0$, $P_{r}=0.71, S_{c}=0.22, R=1.0, Q_{H}=0.5, k_{r}=0.5, K=1.0, M=2.0, \Omega=1.0$ at $t=1.0$.

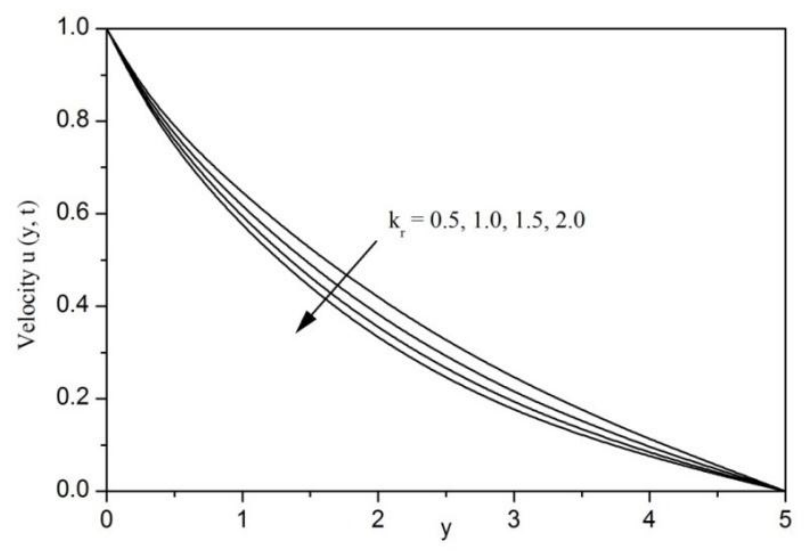

Fig. 11: Effect of chemical reaction rate $k_{r}$ on the fluid velocity when $G_{r}=2.0, G_{m}=2.0$, $P_{r}=0.71, S_{c}=0.22, R=1.0, Q_{H}=0.5, S_{r}=1.0, K=1.0, M=2.0, \Omega=1.0$ at $t=1.0$.

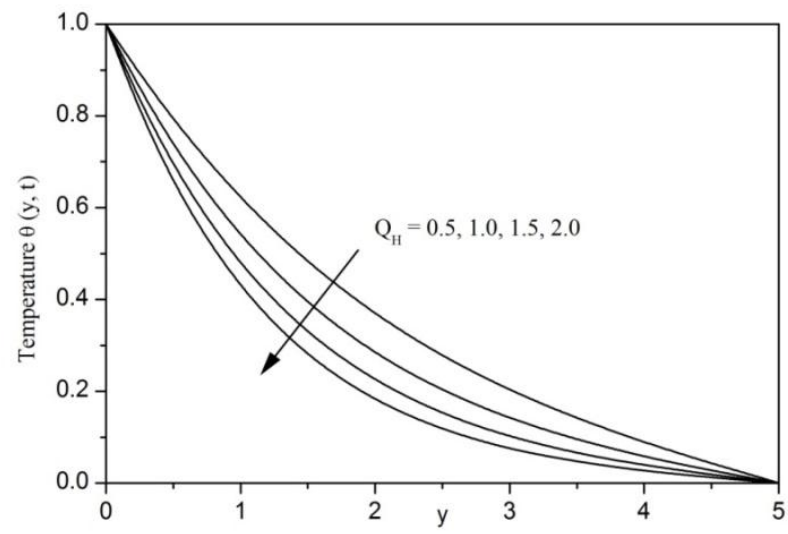

Fig. 12: Effect of heat absorption parameter $Q_{H}$ on the fluid temperature when $P_{r}=0.71, R=1.0$ at $t=1.0$. 


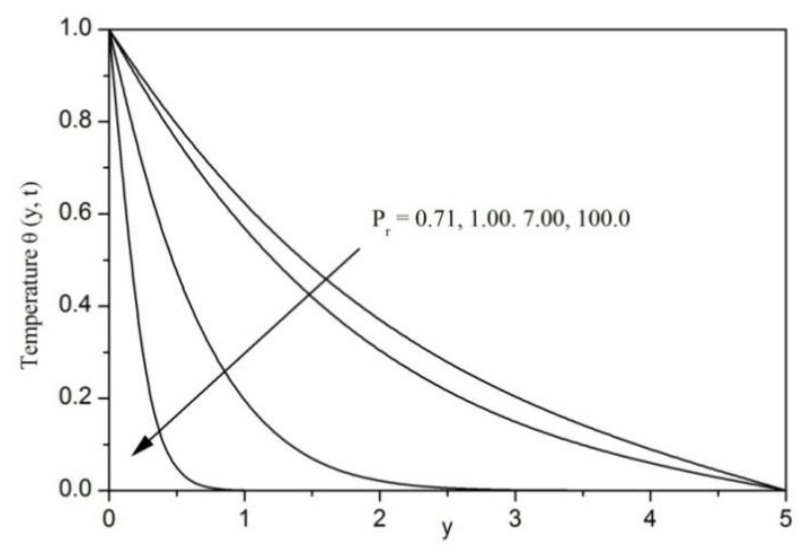

Fig. 13: Effect of Prandtl number $P_{r}$ on the fluid temperature when $R=1.0, Q_{H}=0.5$ at $t=1.0$.

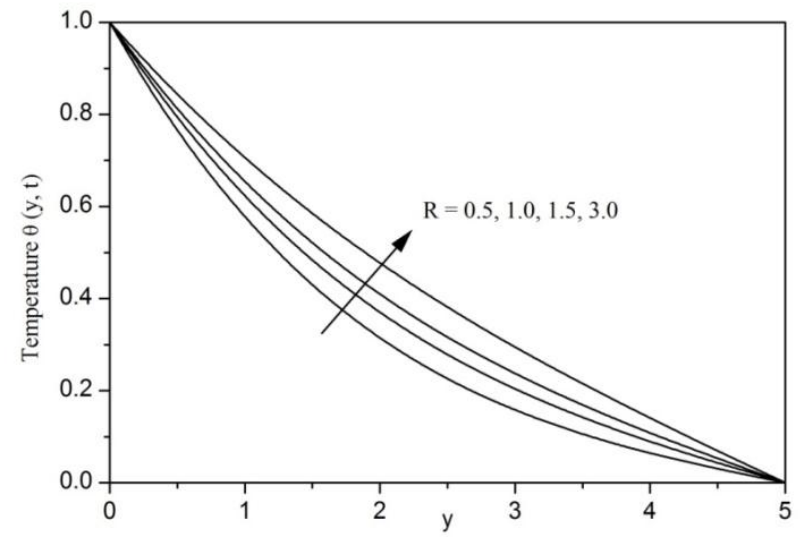

Fig. 14: Effect of radiation parameter $R$ on the fluid temperature when $P_{r}=0.71, Q_{H}=0.5$ at $t=1.0$.

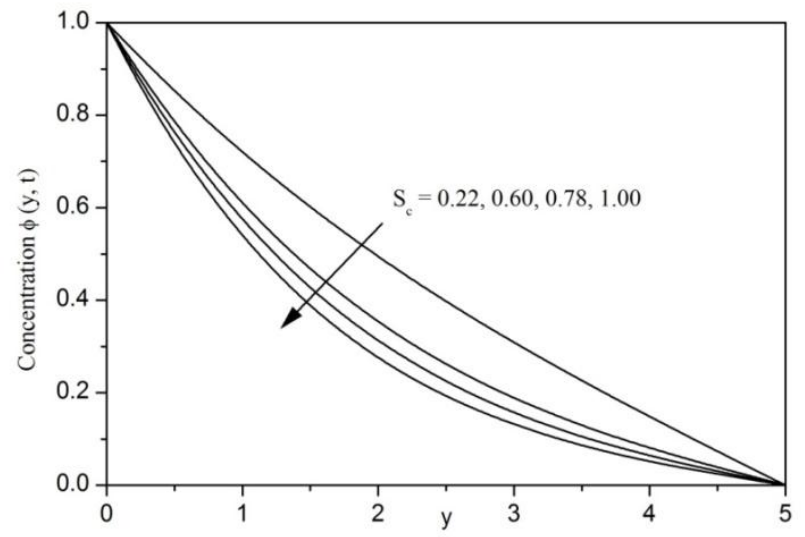

Fig. 15: Effect of Schmidt number $S_{c}$ on the fluid concentration when $P_{r}=0.71, R=1.0, S_{r}=1.0, Q_{H}=0.5, k_{r}=0.5$ at $t=1.0$. 


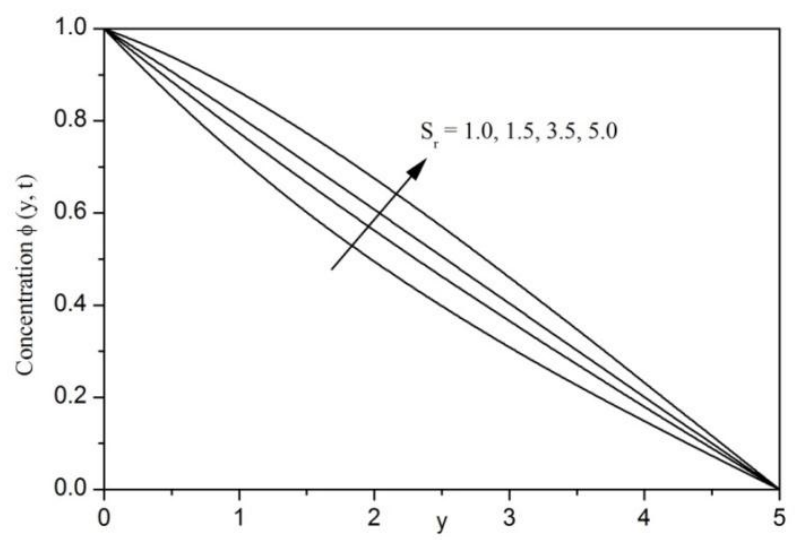

Fig. 16: Effect of Soret number $S_{r}$ on the fluid concentration when $P_{r}=0.71, S_{c}=0.22, R=1.0, Q_{H}=0.5, k_{r}=0.5$ at $t=1.0$.

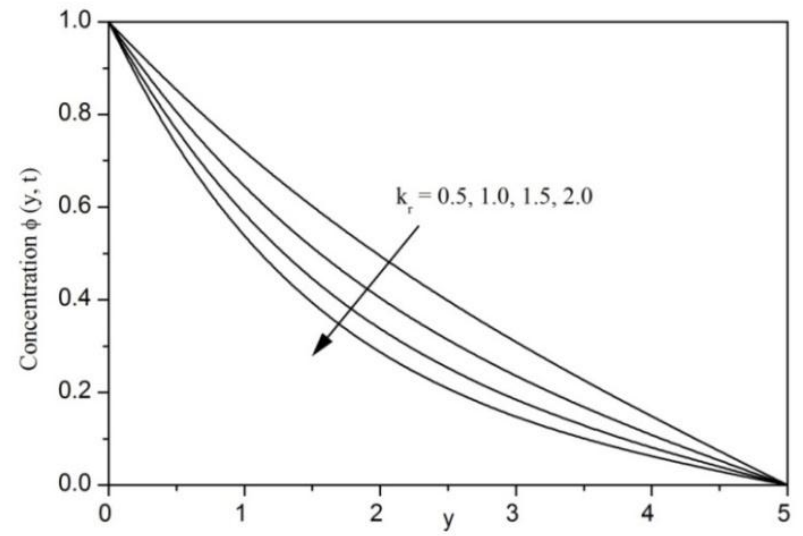

Fig. 17: Effect of chemical reaction rate $k_{r}$ on the fluid concentration when $P_{r}=0.71, S_{c}=0.22, R=1.0, Q_{H}=0.5, S_{r}=1.0$ at $t=1.0$.

Table 1: Effects $P_{r}, Q_{H}, S_{c}, k_{r}, M, R, S_{r}, K, \Omega, G_{r}$ and $G_{m}$ on the skin-friction $(\tau)$.

\begin{tabular}{|c|c|c|c|c|c|c|c|c|c|c|c|}
\hline$P_{r}$ & $Q_{H}$ & $R$ & $S_{c}$ & $k_{r}$ & $S_{r}$ & $M$ & $K$ & $\Omega$ & $G_{r}$ & $G_{m}$ & $\tau$ \\
\hline & & & & & & & & & & & \\
0.71 & 0.5 & 1.0 & 0.22 & 0.5 & 1.0 & 2.0 & 1.0 & 1.0 & 2.0 & 2.0 & 0.244350 \\
7.00 & 0.5 & 1.0 & 0.22 & 0.5 & 1.0 & 2.0 & 1.0 & 1.0 & 2.0 & 2.0 & 0.339542 \\
0.71 & 1.0 & 1.0 & 0.22 & 0.5 & 1.0 & 2.0 & 1.0 & 1.0 & 2.0 & 2.0 & 0.260392 \\
0.71 & 0.5 & 1.5 & 0.22 & 0.5 & 1.0 & 2.0 & 1.0 & 1.0 & 2.0 & 2.0 & 0.237580 \\
0.71 & 0.5 & 1.0 & 0.60 & 0.5 & 1.0 & 2.0 & 1.0 & 1.0 & 2.0 & 2.0 & 0.273632 \\
0.71 & 0.5 & 1.0 & 0.22 & 1.0 & 1.0 & 2.0 & 1.0 & 1.0 & 2.0 & 2.0 & 0.262128 \\
0.71 & 0.5 & 1.0 & 0.22 & 0.5 & 2.5 & 2.0 & 1.0 & 1.0 & 2.0 & 2.0 & 0.230256 \\
0.71 & 0.5 & 1.0 & 0.22 & 0.5 & 1.0 & 2.5 & 1.0 & 1.0 & 2.0 & 2.0 & 0.511804 \\
0.71 & 0.5 & 1.0 & 0.22 & 0.5 & 1.0 & 2.0 & 2.0 & 1.0 & 2.0 & 2.0 & 0.168454 \\
0.71 & 0.5 & 1.0 & 0.22 & 0.5 & 1.0 & 2.0 & 1.0 & 1.5 & 2.0 & 2.0 & 0.142322 \\
0.71 & 0.5 & 1.0 & 0.22 & 0.5 & 1.0 & 2.0 & 1.0 & 1.0 & 4.0 & 2.0 & 0.050864 \\
0.71 & 0.5 & 1.0 & 0.22 & 0.5 & 1.0 & 2.0 & 1.0 & 1.0 & 2.0 & 4.0 & 0.088760 \\
& & & & & & & & & & \\
\hline
\end{tabular}




\section{CONCLUSIONS}

In this paper, the effects of radiation and thermal diffusion on unsteady hydro-magnetic natural convection heat and mass transfer flow of a rotating and chemically reacting fluid past an infinite vertical porous flat plate in the presence heat sink is provided. The dimensionless governing partial differential equations have been solved numerically by using the Ritz finite element method. The significant findings of the study are summarized as follows:

1. Magnetic parameter, Prandtl number, heat absorption parameter, Schmidt number and chemical reaction rate tends to decrease the fluid velocity in the boundary layer.

2. Radiation parameter, permeability parameter, rotation parameter, Soret number, thermal Grashof number and mass Grashof number tends to increase the fluid velocity in the boundary layer.

3. Heat absorption parameter and Prandtl number tends to decrease the fluid temperaturewhereas radiation parameter tends to increase the fluid temperature.

4. Schmidt number and chemical reaction rate tends to decrease the fluid concentration whereas Soret number tends to increase the fluid concentration.

5. The effect of the above stated physical parameters on the skin-friction shows quite the opposite effect to that of the fluid velocity.

\section{REFERENCES}

[1] P. Chandran, N. C. Sacheti and A. K. Singh (1998): Unsteady hydro-magnetic free convection flow with heat flux and accelerated boundary motion, Journal of Physical Society of Japan, 67, 124-129.

[2] R. Muthucumaraswamy, P. Ganesan and V. M. Soundalgekar (2001): Heat and mass transfer effects on flow past an impulsively started vertical plate, Acta Mechanica, 146, No.1-2, 1-8.

[3] R. C. Chaudary and A. Jain (2007): Combined heat and mass transfer effects on MHD free convection flow past an oscillating plate embedded in porous medium, Rom. J. Phys, 52, No.5-7, 505-524.

[4] G. Palani and I. A. Abbas (2009): Free convection MHD flow with thermal radiation from an impulsively started vertical plate, Non Linear Analysis Modelling and Control, 14, No.1, 73-84.

[5] M. Siviah, A. S. Nagarajan and P. S. Reddy (2009): Heat and mass transfer effects on MHD free convection flow past a vertical porous plate, The ICFAI University Journal of Computational Mathematics, 2, No.2, $14-21$.

[6] K. Das and S. Jana (2010): Heat and mass transfer effects on unsteady MHD free convection flow near a moving vertical plate in porous medium, Bull. Soc. Math. BanjaLuka, 17, No.10, 15 - 32 .
[7] M. A. Sattar (1994): Free convection and mass transfer flow through a porous medium past an infinite vertical porous plate with time dependent temperature and concentration, Ind. J. pure and Appl. Math, 23, 759-766.

[8] A. J. Chamkha (2000): Thermal radiation and buoyancy effects on hydro-magnetic flow over an accelerating permeable surface with heat source or sink, Int. J. Engg. Sci, 38, No.15, 1699-1712.

[9] M. Acharya, G. C. Dash and L. P. Singh (2000): Magnetic field effects on free convection and mass transfer flow through porous medium with constant suction and constant heat flux, Ind. J. Pure and Appl. Math, 31, No.1, 1-18.

[10] Y. J. Kim (2001): Unsteady MHD convective flow of polar fluids past a vertical moving porous plate in a porous medium, Int. J. Heat and Mass Transfer, 44, No.15, 2791-2799.

[11] A. J. Chamkha (2004): Unsteady MHD convective heat and mass transfer past a semi infinite vertical permeable moving plate with heat absorption, Int. J. Engg. Sci, 42, No.2, 217-230.

[12] A. N. Donald and A. Bejan (2006): Convection in porous media, Springer Science and Business media.

[13] M. M. Ramana and M. A. Sastry (2006): Magnetohydrodynamic convective flow of a micro-polar past a continuously moving vertical porous plate in the presence of heat generation/absorption, J. Heat Transfer, 128, No.2, 142-152.

[14] V. S. Rajesh and V. K. Varma (2010): Heat source effects on MHD flow past an exponentially accelerated vertical plate with variable temperature through a porous medium, Int. J. Appl. Math. Mech, 6, No. 12, 68-78.

[15] R. Kandasamy, K. Periasamy and K. K. S. Prabhu (2005): Effects of chemical reaction on heat and mass transfer along a wedge with heat source and convection in the presence of suction or injection, Int. J. Heat and Mass Transfer, 48, 1288-1304.

[16] M. A. Seddeek, A. A. Darwish and M. S. Abdelmeguid (2007): Effects of chemical reaction and variable viscosity on hydro-magnetic mixed convection heat and mass transfer for hiemenz flow through porous media with radiation, Communication in Non-Linear Science and Numerical Simulation, 12, No.2, 195 - 213.

[17] R. Muthucumaraswamy and B. Janakiraman (2008): Mass transfer effects on isothermal vertical oscillating plate in the presence of chemical reaction, Int. J. Appl. Math and Mech, 4, 66-74.

[18] A. Mahdy (2010): Effect of chemical reaction and heat generation/absorption on double diffusive convection from a vertical truncated cone in porous 
media with variable viscosity, Int. Comm. in Heat and Mass Transfer, 37, No. 5, 548-554.

[19] S. M. Alharbi, M. A. A. Bazid and M. S. Et. Gendy (2010): Heat and mass transfer in MHD viso-elastic fluid through a porous medium over a stretching sheet with chemical reaction, Applied Mathematics, 1, No.66, 446.

[20] N. Senapati and R. K. Dhal (2011): Magnetic field effect on heat and mass transfer of hydro-magnetic flow past a vertical oscillating plate in the presence of chemical reaction, AMSE, B-2, 79, 60-66.

[21] D. V. Sekhar and G. Viswanath Reddy (2012): Effects of chemical reaction on MHD free convection oscillatory flow past a porous plate with viscous dissipation and heat sink, Advance in Applied Science Research, 3, 3206-3215.

[22] H. L. Agarwal, P. C. Ram and V. Singh (1984): Effects of Hall current on hydro-magnetic free convection with mass transfer in a rotating fluid, Astrophysics Space Science, 100, 297-283.

[23] A. R. Bestman and S. K. Adjepong (1998): Unsteady hydro-magnetic free convection flow with radiative heat transfer in a rotating fluid, Astrophysics Space Science, 143, 217-224.

[24] G. E. A. Azzam (2002): Radiation effects on the MHD mixed free-fixed convection flow past a semiinfinite moving vertical plate for high temperature differences, Phys. Scripta, 66, 71-76.

[25] C. I. Cookey, A. Ogulu and V. B. Omubo-Pepple (2003): Influence of viscous dissipation and radiation on unsteady MHD free convection flow past an infinite heated vertical plate in a porous medium with time dependent suction, Int. J. Heat and Mass Transfer, 46, 2305-2311.

[26] I. U. Mbeledogu and A. Ogulu (2007): Heat and mass transfer of an unsteady MHD natural convection flow of a rotating fluid past a vertical porous flat plate in the presence of radiative heat transfer, Int. J. Heat and Mass Transfer, 50, 19021908 .

[27] R. T. Shanker and R. M. Gnaneshwar (2007): Radiation effects on MHD flow past an impulsively started infinite vertical plate through a porous medium with variable temperature and mass diffusion, Journal of Pure and Applied Physics, 19, No.3, 191-200.

[28] M. A. A. Mahmoud (2009): Thermal radiation effects on unsteady MHD free convection flow past a vertical plate with temperature dependent suction, Canadian J. Chem. Engg, 87, 47-52.

[29] N. Ahmed and H. K. Sarmah (2009): Thermal radiation effects on a transient MHD flow with mass transfer past an impulsively fixed infinite vertical plate, Int. J. Appl. Math and Mech, 5, No.5, 87-98.
[30] S. Mukhopadhyay (2009): Effects of thermal radiation on unsteady mixed convection flow and heat transfer over a porous stretching surface in a porous medium, Int. J. Heat and Mass Transfer, 52, 3261-3265.

[31] S. Sharma and R. K. Deka (2012): Thermal radiation and oscillating plate temperature effects on unsteady MHD flow past a semi infinite porous vertical plate in the presence of chemical reaction, Int. J. Phys and Math. Sci, 2, No.2, 33-52.

[32] M. Muralidharan and R. Muthucumaraswamy (2013): Radiation effects on linearly accelerated plate with variable mass diffusion in the presence of magnetic field, Appl. Math. Sci, 7, No.113, 56455656. 